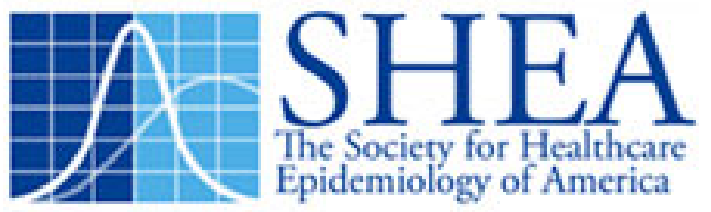

Efficacy of a Novel Light-Activated Antimicrobial Coating for Disinfecting Hospital Surfaces Author(s): Salim Ismail, BSc; Stefano Perni, PhD; Jonathan Pratten, PhD; Ivan Parkin, PhD; Michael Wilson, DSc

Reviewed work(s):

Source: Infection Control and Hospital Epidemiology, Vol. 32, No. 11 (November 2011), pp. 1130-1132

Published by: The University of Chicago Press on behalf of The Society for Healthcare Epidemiology of America

Stable URL: http://www.jstor.org/stable/10.1086/662377

Accessed: 19/09/2012 06:48

Your use of the JSTOR archive indicates your acceptance of the Terms \& Conditions of Use, available at http://www.jstor.org/page/info/about/policies/terms.jsp

JSTOR is a not-for-profit service that helps scholars, researchers, and students discover, use, and build upon a wide range of content in a trusted digital archive. We use information technology and tools to increase productivity and facilitate new forms of scholarship. For more information about JSTOR, please contact support@jstor.org. 


\section{Efficacy of a Novel Light-Activated Antimicrobial Coating for Disinfecting Hospital Surfaces}

\author{
Salim Ismail, BSc; ${ }^{1}$ Stefano Perni, $\mathrm{PhD} ;^{1}$ \\ Jonathan Pratten, $\mathrm{PhD} ;^{1}$ Ivan Parkin, $\mathrm{PhD} ;{ }^{2}$ \\ Michael Wilson, DSc ${ }^{1}$
}

Silicone polymers containing the light-activated antimicrobial agent methylene blue with or without gold nanoparticles were evaluated for their ability to reduce the microbial load on surfaces in a clinical environment. When irradiated with white light, polymers containing nanogold were more effective in this respect than those containing only methylene blue.

Infect Control Hosp Epidemiol 2011;32(11):1130-1132

Nosocomial infections pose a significant threat to hospital patients, staff, and visitors. Clinical environments often harbor pathogens that may then be transmitted to healthcare workers and patients. The use of light-activated antimicrobial agents (photosensitizers) embedded in polymers is a novel approach that may be used for decontaminating surfaces. ${ }^{1}$ Recently, the incorporation of gold $(\mathrm{Au})$ nanoparticles into these polymers has been shown to improve their antimicrobial effectiveness in vitro. ${ }^{2}$ Here, we show that silicone polymers containing the photosensitizer methylene blue (MB) and $\mathrm{Au}$ nanoparticles can be used to reduce environmental contamination in a dental clinic and that high numbers of methicillin-resistant Staphylococcus aureus (MRSA) can be killed under similar lighting conditions.

\section{METHODS}

Silicone polymers. Silicone polymers were prepared as described elsewhere. ${ }^{2}$

Light source. The light source used was a General Electric 28-W Starcoat BIAX 2D T5 compact fluorescent lamp (GE Lighting). This was suspended between clamp stands atop a shelving unit $35 \mathrm{~cm}$ above the base of the stands. ${ }^{3}$ Ambient clinical lights were kept on during each experiment. For laboratory experiments, the light source was fixed in a refrigerated incubator at $22^{\circ} \mathrm{C}$. Light intensity during experiments was measured using a digital luxmeter.

Clinical testing. Clinical testing was conducted as described elsewhere. ${ }^{3}$

Laboratory testing. MRSA strain eMRSA- $16^{4}$ was used to determine the ability of the polymers to kill a clinically relevant organism. eMRSA-16 was cultured in brain-heart infusion broth for 16 hours at $37^{\circ} \mathrm{C}$. This was diluted to give a suspension containing approximately $10^{8}$ colony-forming units (CFUs)/mL. Triplicate samples of each coating (control, $\mathrm{MB}$, and $\mathrm{MB}+\mathrm{Au}$ ) were placed in a humidity chamber, and each was inoculated with $250 \mu \mathrm{L}$ of the bacterial suspension. The chamber was placed in the incubator for 6 or 24 hours.
The light intensity was similar to that used in the clinic experiments. To assess the effect of each coating in the dark, a duplicate humid chamber was covered with aluminum foil and placed in the incubator for 24 hours. Survivors were enumerated by viable counting as described elsewhere. ${ }^{3}$

Statistical analyses. The effect of coating treatment (control, $\mathrm{MB}$, and $\mathrm{MB}+\mathrm{Au}$ ) on the viable count of microbes was evaluated using repeated-measures (2-way) analysis of variance. For all analyses, differences were considered statistically significant when $P$ was less than .05 .

\section{RES U L T S}

The mean light intensity at the surface of the coatings in the clinic was 2,305 lux. The overall mean counts ( \pm standard error of the mean $[\mathrm{SEM}]$ ) of microbes on the settle plates after 48 hours were $3.49 \pm 0.35 \mathrm{CFUs} / \mathrm{cm}^{2}$ (anaerobic cultivation) and $5.04 \pm 0.28 \mathrm{CFUs} / \mathrm{cm}^{2}$ (aerobic cultivation). Control silicone coatings showed a higher microbial load, with overall mean counts $( \pm$ SEM) of $8.23 \pm 2.42$ CFUs/ $\mathrm{cm}^{2}$ (anaerobic cultivation) and $13.80 \pm 1.95 \mathrm{CFUs} / \mathrm{cm}^{2}$ (aerobic cultivation). For anaerobically cultivated microbes, silicone coatings containing $\mathrm{MB}$ and those containing $\mathrm{MB}+$ Au showed $51.3 \%$ and $71.2 \%$ mean reductions in the recovery of viable microbes, respectively, compared with control coatings (Figure 1). The number of aerobically cultivated microbes was reduced by $32.7 \%$ and $54.8 \%$, respectively, on coatings containing $\mathrm{MB}$ and those containing $\mathrm{MB}+\mathrm{Au}$. For $\mathrm{MB}+\mathrm{Au}$ coatings, these reductions were significant for both aerobic $(P=.001)$ and anaerobic $(P=.014)$ microbes. The reduction observed on coatings containing only $\mathrm{MB}$ was at an intermediary level that was not significantly different from the reduction observed on control coatings or on those containing $\mathrm{MB}+\mathrm{Au}$, for both aerobically $(P=.063$ and $P=$ .348 , respectively) and anaerobically $(P=.546$ and $P=$ .358 , respectively) cultivated microbes.

The overall mean light intensities ( \pm SEM) for the 6- and 24-hour irradiation experiments with MRSA were $2,368.8 \pm 22.41$ and $2,102.4 \pm 16.59$, respectively. When irradiated for 24 hours, silicone coatings containing $\mathrm{MB}$ and those containing $\mathrm{MB}+\mathrm{Au}$ achieved substantial reductions of $99.33 \%$ and $99.99 \%$, respectively, compared with control coatings (Figure $2 A$ ). This level of killing was highly significant for both coating types $(P<.001)$. There was no significant difference in the level of killing observed between coatings containing $\mathrm{MB}$ and those containing $\mathrm{MB}+\mathrm{Au}$ over 24 hours $(P=.381)$. When control, $\mathrm{MB}$, and $\mathrm{MB}+\mathrm{Au}$ polymers were kept in the dark, increases of $133 \%, 204 \%$, and $215 \%$, respectively, in the viable count were found. When the irradiation time was reduced to 6 hours (Figure $2 B$ ), coatings containing $\mathrm{MB}$ and those containing $\mathrm{MB}+\mathrm{Au}$ reduced the viable count by $56.51 \%$ and $92.30 \%$, respectively, compared 
with control coatings. This reduction was highly significant for both coating types $(P=.006$ for $\mathrm{MB}$ and $P<.001$ for $\mathrm{MB}+\mathrm{Au}) \cdot \mathrm{MB}+\mathrm{Au}$ polymers showed a further reduction of $82.29 \%$ of viable microbes detected on coatings containing $\mathrm{MB}$ and a significant enhancement $(P=.003)$ in the reduction of total viable MRSA by $35.79 \%$ compared with MB coatings.

\section{I S C USSI ON}

The results of this study show that silicone polymers containing $\mathrm{MB}$ and 2-nm gold nanoparticles are able to reduce the microbial load in a clinical environment when irradiated with white light at an intensity similar to that encountered adjacent to a patient during treatment as well as in certain other areas of hospitals. Regulations specify minimum light intensities of 1,000 lux for accident and emergency departments, 10,000 lux for pathology laboratories, and up to 100,000 lux for operating theaters. ${ }^{5}$ Polymers containing only MB were less effective than those that also contained gold nanoparticles, as the latter achieved statistically significant reductions of $71 \%$ and $55 \%$ for anaerobically and aerobically cultivable microbes, respectively. It was of interest to determine the ability of the polymers to kill very high numbers of a clinically important bacterium, MRSA, under lighting conditions similar to those used in clinical testing. When exposed to white light of more than 2,000 lux, silicone poly-

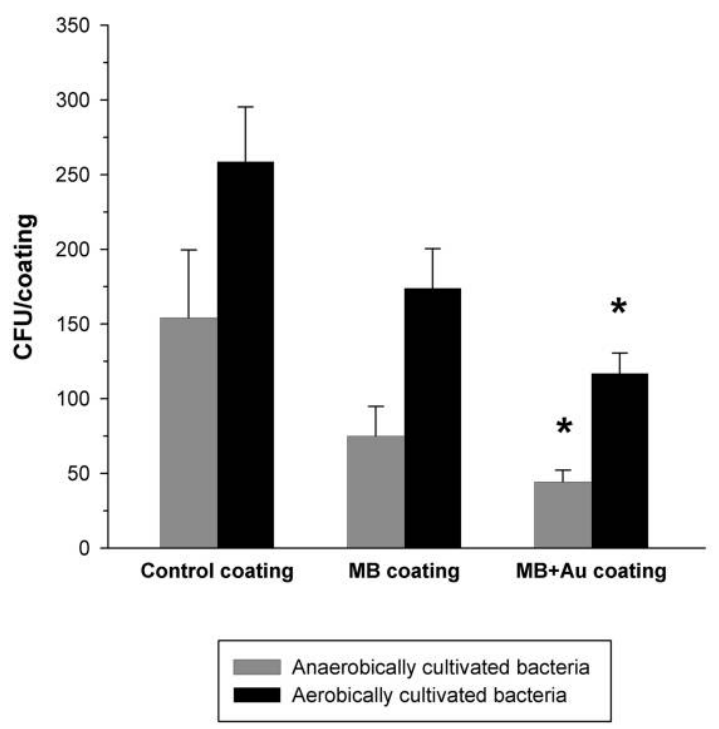

FIGURE 1. Recovery of viable microbes from the surface of silicone elastomer samples placed in a dental clinic for 48 hours and exposed to light from a $28-\mathrm{W}$ fluorescent lamp. Bars represent the mean number of colony-forming units (CFUs; \pm standard error of the mean) per silicone coating for aerobically and anaerobically cultivated microbes. Results are from 20 independent experiments performed in duplicate. An asterisk $(*)$ indicates that the reduction in the recovery of viable microbes is significantly different $(P<.05)$ from that for the control coating. $\mathrm{MB}$, methylene blue; $\mathrm{Au}$, gold.
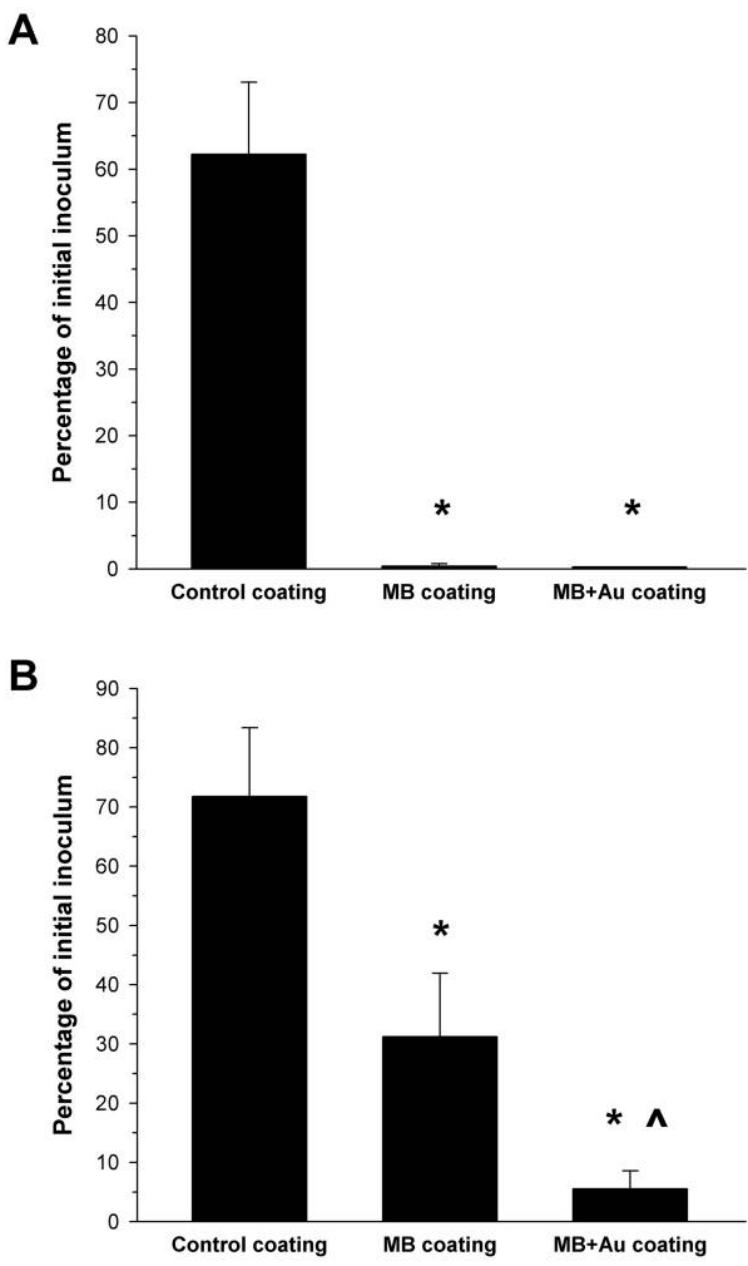

FIGURE 2. Survival of methicillin-resistant Staphylococcus aureus (MRSA) suspensions on the surface of silicone elastomers exposed to $28-\mathrm{W}$ fluorescent light. $A$, Bars represent the mean number of MRSA ( \pm standard error of the mean [SEM]) recovered after a 24hour incubation $\left(22^{\circ} \mathrm{C}\right)$, expressed as a percentage of the initial MRSA inoculum at time zero. Results are from 3 independent experiments performed in triplicate. An asterisk $(*)$ indicates that the reduction in the recovery of viable bacteria is significantly different $(P<.05)$ from that for the control coating. $B$, Bars represent the mean number of MRSA $( \pm$ SEM) recovered after a 6-hour incubation $\left(22^{\circ} \mathrm{C}\right)$, expressed as a percentage of the initial MRSA inoculum at time zero. Results are from 5 independent experiments performed in triplicate. An asterisk $(*)$ indicates that the reduction in the recovery of viable bacteria is significantly different $(P<.05)$ from that for the control coating, and a caret $(\wedge)$ indicates that the enhancement in the reduction of viable MRSA is significantly different $(P<.05)$ from that for the MB coating.

mers containing $\mathrm{MB}$ and those containing $\mathrm{MB}+\mathrm{Au}$ inoculated with $10^{8}$ CFUs of MRSA were able to achieve reductions of more than $99 \%$ in the viable count over 24 hours. This confirms the potential for these types of polymers to be useful antimicrobial coatings. Although there was no difference in the MRSA-killing ability of MB polymers and 
$\mathrm{MB}+\mathrm{Au}$ polymers over 24 hours, over a 6-hour period the $\mathrm{MB}+\mathrm{Au}$ polymers were more effective than MB polymers at reducing MRSA numbers.

When tested in a dental clinic, the coatings appeared to be less effective at reducing microbial numbers than they were under laboratory conditions. However, the clinical setting is a diverse environment with multiple factors that contribute to microbial flux at any given time. The amount and type of clinical activity taking place, the level of air flow, and the prevailing ambient temperature all contribute to the extent of the microbial load. In such an environment, there will likely be continual arrival of new microbes on surfaces. Given that there is likely to be a lag between the arrival of a microbe on a surface and light-activated killing, it is possible that microbes settling on coating surfaces immediately before removal from the clinic were not killed because of insufficient time to enable lethal photosensitization. Additionally, some microbial species are more difficult to kill than others-for example, gram-positive bacteria are more susceptible than gram-negative bacteria. ${ }^{6}$ Similarly, the liquid matrix in which microbes in clinical environments are suspended (saliva, serum) has an effect on microbial survival and on the effectiveness of lethal photosensitization. ${ }^{7}$ Therefore, it is unlikely that antimicrobial-coated surfaces would be completely free of microbes at any given time.

In this study, we have demonstrated the ability of MBembedded silicone polymers to reduce the microbial load in a clinic. We have also shown that the incorporation of $2-\mathrm{nm}$ gold nanoparticles into these polymers enhances the reduction in microbial load. This is the first report, to our knowledge, of the effectiveness of such nanogold-containing polymers under fluorescent lighting in a clinical environment.

\section{ACKNOWLEDGMENTS}

We thank Elizabeth Watts for allowing us the use of a dental clinic at the Eastman Dental Hospital and Dr. Aviva Petrie for assistance with statistical analyses. I.P. thanks the Wolfson/Royal Society for a merit award.

Financial support. This work was funded by a grant from the Higher Education Funding Council for England.
Potential conflicts of interest. M.W.'s research group has received funding for research on light-activated antimicrobial agents from Ondine Biomedical, Vancouver, Canada; he also holds stock options in this company, which licenses from his employer (University College London) patents relating to the use of light-activated antimicrobial agents. All other authors report no conflicts of interest relevant to this article.

Affiliations: 1. Division of Microbial Diseases, UCL Eastman Dental Institute, University College London, London, United Kingdom; 2. Department of Chemistry, University College London, London, United Kingdom.

Address correspondence to Michael Wilson, DSc, Division of Microbial Diseases, UCL Eastman Dental Institute, University College London, 256 Gray’s Inn Road, London WC1X 8LD, United Kingdom (mike.wilson@ucl .ac.uk).

Received April 12, 2011; accepted June 30, 2011; electronically published September 20, 2011

(C) 2011 by The Society for Healthcare Epidemiology of America. All rights reserved. 0899-823X/2011/3211-0014\$15.00. DOI: 10.1086/662377

\section{REFERENCES}

1. Decraene V, Pratten J, Wilson M. Cellulose acetate containing toluidine blue and rose bengal is an effective antimicrobial coating when exposed to white light. Appl Environ Microbiol 2006;72: 4436-4439.

2. Perni S, Piccirillo C, Pratten J, et al. The antimicrobial properties of light-activated polymers containing methylene blue and gold nanoparticles. Biomaterials 2009;30:89-93.

3. Decraene V, Pratten J, Wilson M. An assessment of the activity of a novel light-activated antimicrobial coating in a clinical environment. Infect Control Hosp Epidemiol 2008;29:1181-1184.

4. Murchan S, Aucken H, O'Neill GL, et al. Emergence, spread, and characterization of phage variants of epidemic methicillin-resistant Staphylococcus aureus 16 in England and Wales. J Clin Microbiol 2004;42:5154-5160.

5. Chartered Institute of Building Service Engineers. CIBSE Lighting Guide LG2: Hospitals and Healthcare Buildings. 1989.

$\rightarrow$ 6. Usacheva MN, Teichert MC, Biel MA. Comparison of the methylene blue and toluidine blue photobactericidal efficacy against gram-positive and gram-negative microorganisms. Lasers Surg Med 2001;29:165-173.

$\rightarrow$ 7. Decraene V, Pratten J, Wilson M. Novel light-activated antimicrobial coatings are effective against surface-deposited Staphylococcus aureus. Curr Microbiol 2008;57:269-273. 